Review

\title{
The Role of Tumor Associated Macrophages in Hepatocellular Carcinoma
}

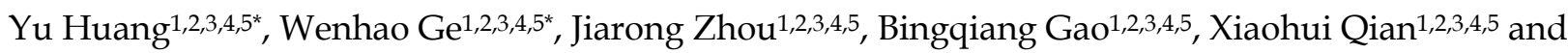 \\ Weilin Wang $1,2,3,4,5 \bowtie$
}

1. Department of Hepatobiliary and Pancreatic Surgery, The Second Affiliated Hospital, Zhejiang University School of Medicine, Hangzhou, Zhejiang 310009.

2. Key Laboratory of Precision Diagnosis and Treatment for Hepatobiliary and Pancreatic Tumor of Zhejiang Province, Hangzhou, Zhejiang 310009.

3. Research Center of Diagnosis and Treatment Technology for Hepatocellular Carcinoma of Zhejiang Province, Hangzhou, Zhejiang 310009.

4. Clinical Medicine Innovation Center of Precision Diagnosis and Treatment for Hepatobiliary and Pancreatic Disease of Zhejiang University, Hangzhou, Zhejiang 310009.

5. Clinical Research Center of Hepatobiliary and Pancreatic Diseases of Zhejiang Province, Hangzhou, Zhejiang 310009.

* These authors contributed equally to this work.

$\square$ Corresponding author: Weilin Wang, Department of Hepatobiliary and Pancreatic Surgery, The Second Affiliated Hospital, Zhejiang University School of Medicine, No. 88 Jiefang Road, Hangzhou, Zhejiang, China, 310009; E-mail: wam@zju.edu.cn; Tel: +86057187783820; Fax: +86057187068001.

(c) The author(s). This is an open access article distributed under the terms of the Creative Commons Attribution License (https://creativecommons.org/licenses/by/4.0/). See http://ivyspring.com/terms for full terms and conditions.

Received: 2020.07.31; Accepted: 2020.12.04; Published: 2021.01.01

\begin{abstract}
Hepatocellular carcinoma ( $\mathrm{HCC})$ is one of the most common cancers worldwide and represents a classic paradigm of inflammation-related cancer. Various inflammation-related risk factors jointly contribute to the development of chronic inflammation in the liver. Chronic inflammation, in turn, leads to continuous cycles of destruction-regeneration in the liver, contributing to HCC development and progression. Tumor associated macrophages are abundant in the tumor microenvironment of HCC, promoting chronic inflammation and HCC progression. Hence, better understanding of the mechanism by which tumor associated macrophages contribute to the pathogenesis of HCC would allow for the development of novel macrophage-targeting immunotherapies. This review summarizes the current knowledge regarding the mechanisms by which macrophages promote $\mathrm{HCC}$ development and progression, as well as information from ongoing therapies and clinical trials assessing the efficacy of macrophage-modulating therapies in HCC patients.
\end{abstract}

Key words: Hepatocellular carcinoma, Tumor associated macrophage, Tumor microenvironment, Immunotherapy

\section{Introduction}

Hepatocellular carcinoma (HCC) is one of the most common cancers worldwide [1]. Despite advances in surgical resection, adjuvant therapy, and liver transplantation during the past decades, the survival rate of HCC patients remains unsatisfactory, with a 5 -year survival rate of approximately $20 \%$. The poor prognosis of HCC is primarily due to its late diagnosis, as well as its high risk of potential recurrence and metastasis [2,3]. Other non-surgical interventions, including transarterial chemoembolization (TACE) and radiofrequency ablation (RFA), can provide disease control only for advanced-stage patients [4]. Systemic administration of sorafenib exerts a weak therapeutic effect in patients with metastatic HCC [5]. Therefore, the development of novel therapeutics with mechanisms different from those of the currently available treatments is crucial to improve the prognosis of HCC patients.

Emerging immunotherapies, including immune checkpoint inhibitors targeting programmed death-1 (PD-1) and cytotoxic T lymphocyte-associated antigen 4 (CTLA4), have revolutionized the therapeutic landscape for various solid tumors [6-9]. HCC is a typical example of inflammation-related cancer. Primary risk factors for HCC include exposure to aflatoxins, alcohol intoxication, non-alcoholic fatty liver disease caused by obesity and metabolic syndrome, as well as viral infections such as hepatitis 
B and C viruses. These factors jointly contribute to hepatic inflammation, fibrosis, and cirrhosis, which are observed in $80 \%$ of HCC patients [10-12]. In the inflammatory tumor microenvironment (TME) of HCC, various non-malignant cells and extracellular matrix components play pivotal roles in cancer development and progression, as well as in resistance to traditional antitumor therapies $[13,14]$.

TME is characterized by infiltration of various non-malignant cells, including stromal cells, fibroblasts, macrophages, endothelial cells, immune cells, and circulating platelets. Cytokines secreted by these cells and the extracellular matrix are also important components of the TME [15-17]. Within the TME, non-malignant cells play crucial roles in promoting cancer cell proliferation, invasion, and metastasis. Among them, immune cells coexist and interact with each other initiating complex pathways and ultimately resulting in tumor progression and drug resistance. Thus, the combination of immunological interventions with conventional therapies might be more effective than monotherapies $[18,19]$. Macrophages are major components of the TME; several tumor-promoting roles have been attributed to these tumor associated macrophages (TAMs) [20-23]. TAMs have been implicated in immune suppression, cancer invasion and metastasis, angiogenesis, maintenance of cancer cell stemness, and drug resistance. Furthermore, high levels of TAMs have been associated with poor prognosis in patients with HCC [24-26]. Therefore, a better understanding of the mechanisms underlying the function of TAMs is necessary for the development of novel TAM-targeting immunological interventions, which may provide promising therapeutic approaches for HCC patients [27-29].

This review summarizes the current knowledge regarding the role of macrophages in the pathogenesis of HCC and provides information regarding the currently available immunotherapies as well as ongoing clinical trials assessing the efficacy of macrophage-modulating therapies in HCC patients.

\subsection{Liver macrophages in homostasis}

As one of the main types of innate immune cells, macrophages serve as the first line of defense against pathogenic insults to the body. Macrophages can be found in all tissues and exhibit exceptionally high plasticity and functional diversity [30-32]. Macrophages are involved in phagocytosis, antigen processing and presentation, and orchestration of the immune system by the release of multiple cytokines, regulating inflammation initiation, progression, and resolution [33,34].

The liver harbours the main part body macrophages and is supervised by myeloid cells including blood monocytes, which scan the liver vasculature and eventually infiltrate into the liver. Under homeostasis conditions, monocyte-derived cells can develop into liver dendritic cells (DCs) or monocyte-derived macrophages (MoMFs), the latter do not contribute to the pool of resident macrophages, termed Kuffer cells. Kuffer cells originate from yolk sac-derived precursors during embryogenesis, forming a self-renewing pool of resident macrophages in the liver and playing essential roles in sustaining hepatic and systematic homeostasis [35]. When activated by danger signals, Kuffer cells can promote chronic liver inflammation by inducing the recruitment of immune cells to the liver, including monocytes, which subsequently derived DCs and MoMFs [36]. To date, no specific markers were proved to distinguish human Kuffer cells from monocyte-derived cells (Figure 1).

Additionally, according to their inflammatory states in response to different environmental stimuli, macrophages can be classified into two main subtypes: the classically activated macrophages (M1 macrophages) and alternatively activated macrophages (M2 macrophages). These two distinct functional phenotypes have contradicting roles in regulating inflammation progression in vitro $[37,38]$. M1 macrophages are primarily induced by microbial components, such as lipopolysaccharides (LPS), or by pro-inflammatory cytokines, including interferon- $\gamma$ $(\mathrm{IFN}-\gamma)$, tumor necrosis factor (TNF), and toll-like receptor (TLR) ligands. M1 macrophages exert pro-inflammatory functions by releasing nitric oxide (NO), reactive oxygen species (ROS), and the pro-inflammatory cytokines interleukin (IL)-1, IL-6, IL-12, TNF-a, CXCL5, and CXCL8-10, involving in antigen processing and presentation, promoting the function of effector T cells $[39,40]$. Polarization to the M2 phenotype is induced by IL-4, IL-10, and IL-13, as well as by glucocorticoids. M2 macrophages exert immunosuppressive functions and promote tissue repair by secreting IL-10 and other immunosuppressive cytokines [41,42] (Figure 1). However, there are almost none of the purely M1 or M2 macrophages in scenarios like tumor models or autoimmune disease in vivo. Thus, more research for a better understanding of the relationship between mechanisms and the subphenotypes of macrophages is urgently needed in developing novel therapies. 


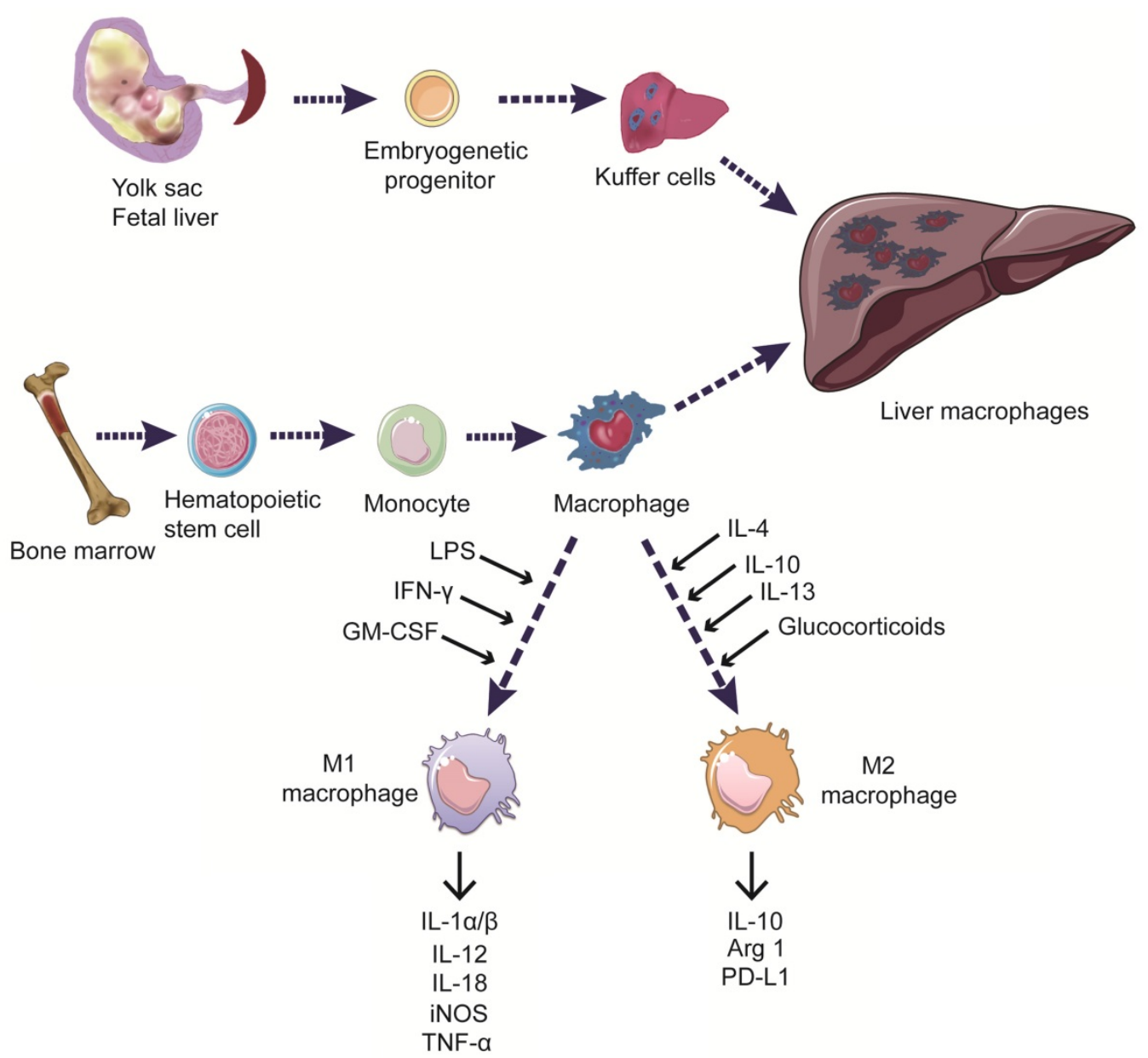

Figure 1. Origin and classification of liver macrophages. Liver macrophages, consisting of Kuffer cells and monocyte-derived macrophages, play essential roles in sustaining hepatic and systematic homeostasis. Myeloid cells including blood monocytes, scan the liver vasculature and eventually derive macrophages infiltrating into the liver. Kuffer cells are resident phagocytes originating from yolk sac-derived precursors during embryogenesis. Macrophages can be classified into two distinct functional phenotypes according to their responses to different environmental stimuli in vitro. MI macrophages are induced by LPS, IFN-y, and GM-CSF. They secrete the pro-inflammatory cytokines IL-1 / / $\beta$, IL-12, IL-18, iNOS, and TNF- $\alpha$, as well as promoting the function of effector T cells. M2 macrophages are induced by IL-4, IL-10, IL-13, and glucocorticoids, and exert anti-inflammatory and immune-regulatory effects by expressing IL-10, Argl, and PD-L1.

\section{Liver macrophages in pathogenesis of HCC}

Increased infiltration of macrophages is a common characteristic of various solid tumors. Concurrently, HCC represents a classic paradigm of inflammation-related cancer. Various inflammationrelated risk factors jointly contribute to the development of chronic inflammation in the liver. Chronic inflammation, in turn, leads to continuous cycles of destruction-regeneration in the liver, contributing to fibrosis and cirrhosis, and eventually development and progression of HCC. Liver macrophages are regulators of this process [43,44]. An immunogenic analysis using patient data from The Cancer Genome Atlas indicated that TAMs are abundant in HCC, which are mostly polarized towards the M2 phenotype. CD68 is commonly used as an indicator of liver TAMs, and the expression levels of CD86 (M1), CD163 (M2), and CD206 (M2) are widely accepted to differentiate between M1 and M2 macrophages in vitro [45]. Low levels of $\mathrm{CD}^{2} 6^{+} \mathrm{M} 1$ macrophages and high levels of $\mathrm{CD}_{206}{ }^{+}$M2 macrophages have been associated with an aggressive phenotype in HCC, suggesting that conjoint analysis of CD86 and CD206 expression may provide a prognostic tool for HCC [46]. Numerous chemokines (CCL2, CCL5, CCL15, CCL20), cytokines (such as CSF-1) and other products of the complement cascade were demonstrated to participate in the mechanism of monocyte-derived macrophages recruitment and migration [23,47-49]. Additionally, several researches recently provide evidence in the transition from Kuffer cells to TAM pool by triggering Her2/Neu pathway [50,51]. Other factors, including mitochondrial DNA (mtDNA), osteopontin (OPN), micro RNAs (miRNAs), circular RNAs (circRNAs) and HCC cell-derived exosomes were also reported to play important roles in TAM recruitment [52-55]. TAMs induce the expression of ST18 in epithelial cells, promoting mutual epithelial cell-macrophage 
dependency in HCC [56]. TAMs also release various immunosuppressive chemokines and cytokines, including IL-10, transforming growth factor beta (TGF- $\beta$ ), which exert immunoregulatory roles. Furthermore, TAMs have been shown to recruit regulatory $\mathrm{T}$ cells (Tregs) to the tumor; the recruitment of Tregs impairs the activation and function of effector T cells [57].

Recent studies have indicated that insulin-like growth factor (IGF)-1 and IGF-2 remodel macrophages during their maturation [58]. Tricarboxylic acid cycle metabolism plays a key role in the epigenetic remodeling of macrophages [59]. In mouse models of glioblastoma, IGF-1 enhanced PI3K-mediated tumor cell proliferation in a macrophage-dependent manner [60]. Nevertheless, the molecular mechanisms underlying the IGF-mediated reprogramming of macrophages in human HCC require further investigation.

Increased levels of TAMs have been shown to promote angiogenesis, cancer cell proliferation, invasion, and metastasis; high levels of TAMs have also been associated with a poor prognosis in HCC patients $[61,62]$. The mechanisms of TAMs in the pathogenesis of HCC are summarized hereinbelow (Figure 2).

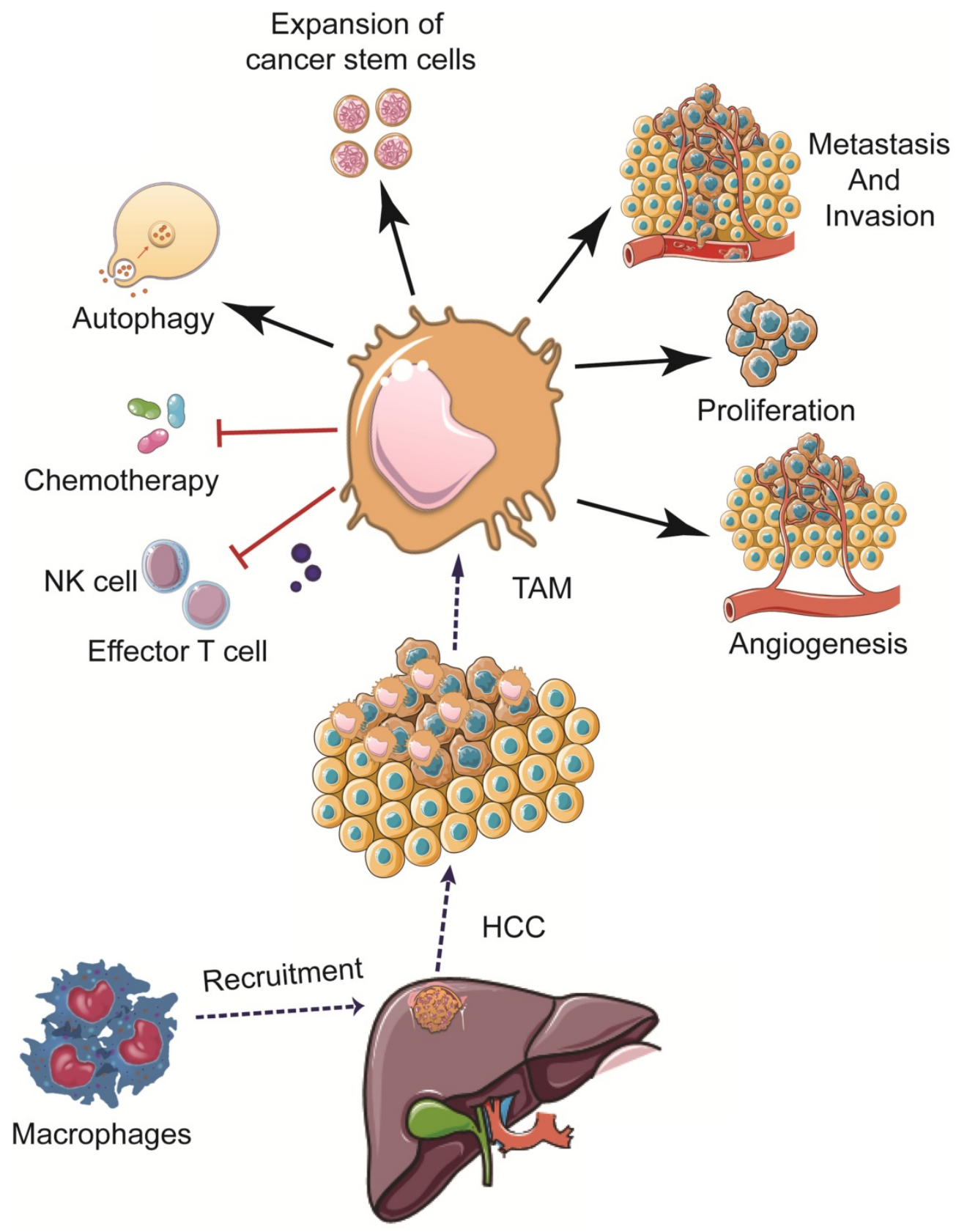

Figure 2. Effects of TAMs in the pathogenesis of HCC. TAM can promote HCC development and progression via multiple mechanisms, including promoting cancer cell proliferation, stemness, invasion, and metastasis, modulating angiogenesis, autophagy, drug resistance, as well as weakening the functions of NK cells and effector T cells, etc. 


\subsection{TAMs promote cancer cell proliferation, invasion, and metastasis in HCC}

M2 macrophages have been shown to play an essential role in promoting cancer cell migration in HCC via the TLR4/STAT3 signaling pathway [63]. Aberrant activation of the NTS/IL-8 pathway was reported to play a pro-tumorigenic role in the inflammatory microenvironment of $\mathrm{HCC}$, by augmenting M2 macrophage-mediated EMT of cancer cells [64]. CXCL8 produced by activated macrophages promotes HCC progression and metastasis [65]. TIM- 3 augments the TGF- $\beta$ mediated polarization of macrophages toward an M2 phenotype, contributing to the poor prognosis of HCC [66]. IL-6 derived by TAMs has been suggested to promote cancer cell invasion, and metastasis in HCC [67]. SPON2 has been reported to promote the infiltration of $\mathrm{M} 1$ macrophages in HCC and suppress metastasis via the integrin/RhoGTPase/Hippo pathway [68]. MicroRNAs, as a class of small non-coding RNAs, regulating gene expression at the post-transcriptional level, were reported to mediate tumor-promoting effects of TAMs recently. Downregulation of miR-28-5p expression in HCC samples has been inversely associated with the levels of TAM infiltration and IL-34 expression; IL-34 further promotes TAM infiltration, resulting in a miR-28-5p-IL-34 feedback loop, which plays an important role in HCC metastasis [69]. MiR-98 has been shown to suppress cancer cell invasion in HCC by promoting macrophage polarization from the M2 to M1 phenotype [70]. MiR-146a-5p, enriched in HCC cell-derived exosomes, was demonstrated to promote infiltration of M2 TAMs, which results in $\mathrm{T}$ cell exhaustion and HCC progression [54]. Coincidentally, long non-coding RNAs also play an important role in the tumor-promoting effects of macrophages in HCC. Long non-coding RNA COX-2 suppresses immune evasion and metastasis in HCC by inhibiting macrophage polarization into an M2 phenotype [71]. Recently, using single-cell RNA sequencing, RIPK1 was demonstrated to induce $\mathrm{CCR}^{+}$macrophages infiltration, as well as promoting liver fibrosis and hepatocarcinogenesis [72]. Wu et al. recently manifested that CD11b/CD18, as well as integrin, which derived from M2 macrophage exosomes, had the potency to boost the migratory potential of HCC cells [73]. Additionally, HCC cell-derived Wnt ligands stimulate M2 polarization of TAMs, which reversely result in tumor growth, metastasis and immunosuppression in HCC [74]. Heat shock transcription factor 1 (HSF1) was demonstrated as a vital mediator in metabolic alteration of HCC cells in cross-talking with TAMs [75].
Hypoxia has been demonstrated to mediate the effects of macrophages, playing an important role in HCC, among other solid tumors. Hypoxia-induced EMT increased the expression of CCL20 in hepatoma cells, leading to indoleamine 2,3-dioxygenase (IDO) upregulation in monocytes-derived macrophages. Macrophages that are derived from IDO+ monocytes counteract effector $\mathrm{T}$ cells and promote tolerance to tumor antigens [76]. Zhang et al. found that the necrotic debris from cancer cells in the hypoxic and inflammatory HCC microenvironment enhanced the release of IL-1 $\beta$ by M2 macrophages. IL-1 $\beta$, in turn, unregulated the expression of HIF-1a in HCC cells in a cyclooxygenase-2-dependent manner, constituting a positive feedback loop promoting EMT in HCC cells [77]. Wu et al. confirmed that TREM-1+ TAMs promote the recruitment of $\mathrm{CCR}^{+}{ }^{+} \mathrm{Foxp}^{+}$Tregs via the ERK/NF-kB pathway, conferring resistance to programmed cell death ligand 1 (PD-L1) treatment in HCC [78].

\subsection{TAMs promote angiogenesis in HCC}

TAMs have been demonstrated to produce numerous angiogenic factors, including vascular endothelial growth factor, platelet-derived growth factor, and several matrix metalloproteinases [79]. The $\mathrm{CCR}^{+}$TAM subset was reported to be highly enriched in highly vascularized HCC tumors and was suggested to drive angiogenesis and tumor vascularization in fibrotic livers [80]. A recent study showed that HCC patients with high serum levels of IL-23 derived from macrophages had tumors with enhanced vascularization [81]. CXCR4, expressing on endothelial cells, which is upregulated by inflammatory cytokines derived from TAMs via ERK pathway activation, was identified as a novel vascular marker in HCC tissues [82]. The combination of sorafenib and zoledronic acid (ZA) has been shown to exert synergistic antitumor effects mediated by downregulation of CXCR4 expression [83].

\subsection{TAMs promote cancer cell stemness in HCC}

Previous studies have suggested that the high heterogeneity and malignancy of HCC are partly attributed to cancer stem cells (CSCs), which promote tumor recurrence, metastasis, and development of resistance to therapies [84]. Numerous cell surface proteins, including EPCAM, CD133, CD124, CD44, and CD90, have been identified as CSC markers $[85,86]$. TAMs have been suggested to promote CSC-like properties via various signaling pathways. Importantly, TAMs facilitated the expansion of stem cells via the IL-6/STAT3 pathway in HCC patients [87]. Fan et al. confirmed that TAMs promote CSC 
properties via TGF- $\beta$ signaling pathway [88]. TNF- $\alpha$ promotes EMT and cell stemness in HCC by activating the $W n t / \beta$-catenin signaling pathway, which can be partially reversed by the Wnt/ $\beta$-catenin inhibitor ICG-001 [89]. In addition, exosomes from TAMs promote cancer cell proliferation and stem cell properties in HCC. For instance, a low level of $\mathrm{miR}-125 \mathrm{a} / \mathrm{b}$ in exosomes from TAMs was shown to inhibit CSCs by targeting CD90 in HCC [90].

\subsection{TAMs promote autophagy in HCC}

Mounting evidence suggests the importance of autophagy in the regulation of the function of TAMs and antitumor immunity [91]. A recent study showed that autophagy-deficient Kupffer cells promoted liver fibrosis, inflammation, and hepatocarcinogenesis via the mitochondrial reactive oxygen species/NF- $\mathrm{kB} /$ IL-1 $\alpha / \beta$ signaling axis [92]. Chang et al. demonstrated that TLR2 related ligands triggered NFKB RELA cytoplasmic ubiquitination and led to its degradation by SQSTM1/p62-mediated autophagy, promoting M2 polarization in macrophages and immunosuppression in HCC [93]. TLR2 deficiency also resulted in a decrease in macrophage infiltration and inhibition of apoptosis signal-regulating kinase 1 and p38 mitogen-activated protein kinase/NF-kB signaling. Subsequently, TLR2 deficiency led to decreased expression levels of IFN- $\gamma$, TNF- $\alpha$, and IL- $1 \alpha / \beta$, as well as increased cell proliferation and suppressed autophagy and apoptosis in mouse liver cells [94]. Tan et al. revealed that the natural compound baicalin inhibited HCC development and progression by TAM repolarization towards the M1 phenotype via autophagy-associated activation of RelB/p52 [95]. Fu et al. suggested that TAMs induce autophagy in HCC cells, which might contribute to oxaliplatin resistance [24]. These findings reveal new roles of TAMs and autophagy in HCC, which could provide new opportunities for the development of more efficient therapies.

\subsection{TAMs modulate therapeutic resistance in HCC}

The orally administrated multikinase inhibitor sorafenib shows limited efficacy in HCC patients due to the development of intolerance and resistance [96]. TAM has been demonstrated to induce immunosuppression and weaken the efficacy of sorafenib in HCC [97]. Zhou et al. demonstrated that tumor-associated neutrophils enhance the recruitment of Tregs and macrophages to the TME in HCC patients, promoting tumor progression and resistance to sorafenib [98]. Oxaliplatin-based chemotherapies are widely used in patients with advanced HCC. It has been reported that TAMs are important drivers of resistance to oxaliplatin by trigging autophagy and apoptosis evasion in HCC cells [24]. The density of TAMs in HCC samples has been associated with the efficiency of transarterial chemoembolization in HCC [24]. Moreover, M2 macrophages have been reported to promote the development of resistance to sorafenib in HCC by secreting hepatocyte growth factor (HGF) [25]. A recent study showed that sorafenib induces pyroptosis in macrophages and facilitates NK cell-mediated cytotoxicity in HCC, highlighting the importance of TAMs as a therapeutic target in HCC [99].

\section{Macrophage-targeting therapies in HCC}

Increasing evidence suggests the critical roles of TAMs in HCC development and progression. Hence, immunotherapies targeting TAMs have emerged as a promising approach to treat patients with HCC. The current therapeutic strategies targeting TAMs include phagocytosis-promoting therapies, inhibition of monocyte recruitment, elimination of pre-existing TAMs in the tumor tissue, remodeling TAM polarization, and neutralizing pro-tumorigenic factors secreted by TAMs [100,101] (Figure 3). The currently available immunotherapies, as well as ongoing clinical trials involving the use of TAM-targeting immunotherapies in HCC, are summarized in Tables 1 and 2.

\subsection{Phagocytosis-promoting therapies}

CD47, mostly known as a receptor for thrombospondin in human myeloid and endothelial cells, has recently been demonstrated to protect host cells from macrophage-mediated destruction by binding to SIRP1a (SHPS-1) expressed on the surface of macrophages [102]. Neutralizing antibodies against CD47 can enhance macrophage-mediated phagocytosis and activate effector T cells [103,104]. IL-6 secretion by TAMs has been reported to upregulate CD47 expression in HCC cells via the STAT3 signaling pathway. CD47 upregulation has been associated with poor overall survival and recurrence-free survival in HCC patients. Blockage of CD47 enhanced TAM-mediated phagocytosis in the presence of chemotherapeutic agents [105]. Yang et al. demonstrated that the HDAC6/let-7i-5p/TSP1 axis reduced the neoplastic and antiphagocytic properties of HCC cells by targeting CD47, providing a promising therapeutic target for the treatment of HCC [106]. Additionally, the anti-CD47 monoclonal antibody (B6H12) suppressed tumor growth and augmented the efficacy of chemotherapy in HCC $[107,108]$. 


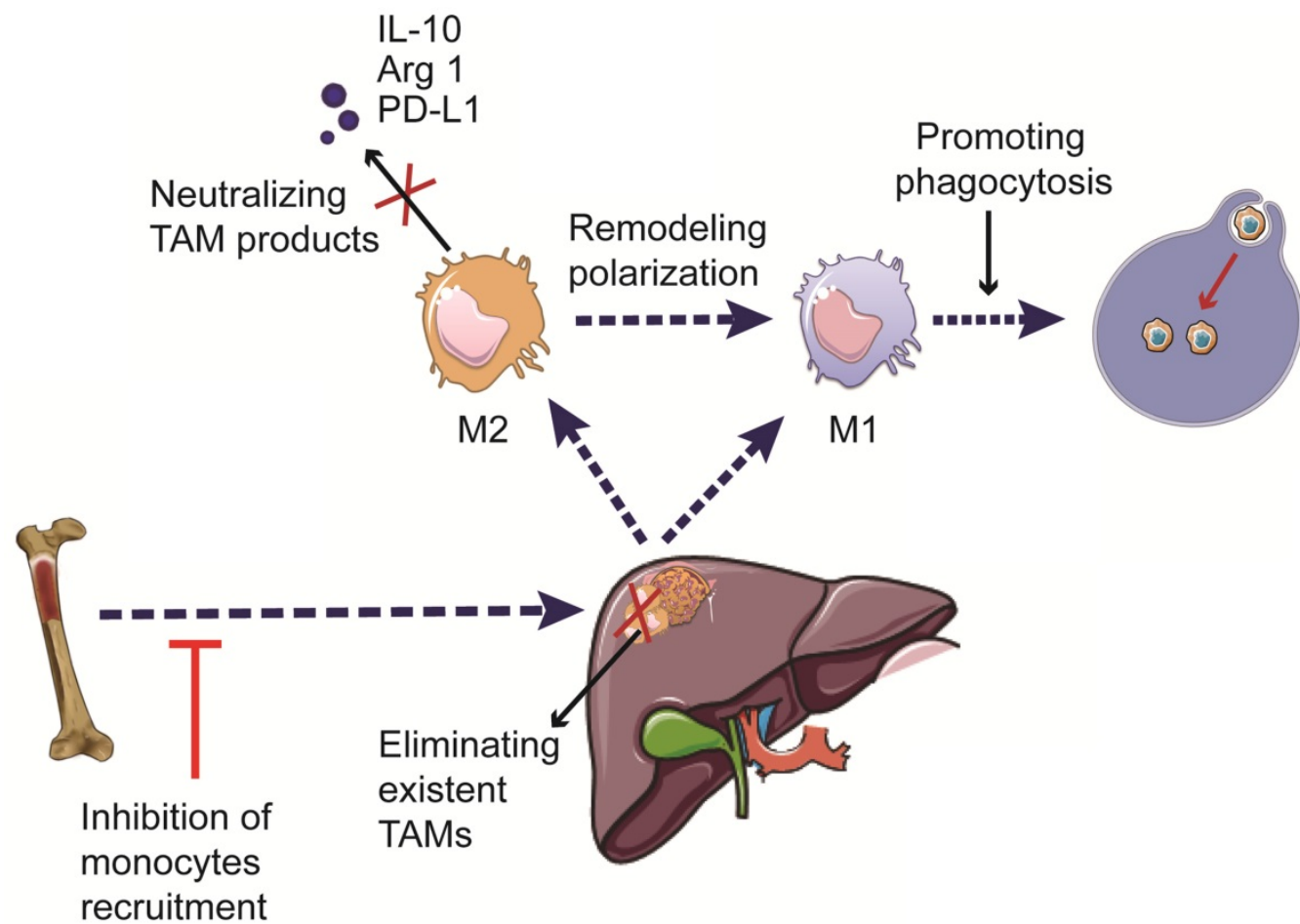

Figure 3. Therapeutic strategies targeting TAMs in HCC. The development of TAM-targeting immunotherapeutic strategies includes induction of phagocytosis, inhibition of monocyte recruitment, elimination of pre-existing TAMs, reprogramming of macrophage polarization, and neutralization of the pro-tumorigenic factors secreted by TAMs.

Table 1. Preclinical agents targeting TAMs for HCC treatment

\begin{tabular}{|c|c|c|c|c|}
\hline Author & Agent & Target & Mechanism of action & Result \\
\hline Lu et al. .107$]$ & Anti-CD47mAbs & CD47 & $\begin{array}{l}\text { Promote phagocytosis of } \\
\text { macrophages }\end{array}$ & $\begin{array}{l}\text { Suppress tumor growth and enhance the effect of chemotherapy } \\
\text { treatment }\end{array}$ \\
\hline Xiao et al.[108] & B6H12 & CD47 & $\begin{array}{l}\text { Promote phagocytosis of } \\
\text { macrophages }\end{array}$ & $\begin{array}{l}\text { Suppress tumor growth and enhance the effect of chemotherapy } \\
\text { treatment }\end{array}$ \\
\hline Li et al. [110] $^{2}$ & RDC018 & CCR2 & Inhibiting monocytes recruitment & Inhibit HCC growth and metastasis, reduce recurrence \\
\hline Yao et al. .97$]$ & 747 & CCR2 & Inhibiting monocytes recruitment & Anti-cancer properties and enhance the efficacy of sorafenib \\
\hline Zhu et al.[113] & GC33 & Glypican-3 & Eliminating existent TAMs & Japanese Phase II study for advanced HCC \\
\hline $\begin{array}{l}\text { Ikeda et } \\
\text { al.[114] }\end{array}$ & GC33 & Glypican-3 & Eliminating existent TAMs & Clinical trail for advanced HCC patients \\
\hline $\begin{array}{l}\text { Zhang et } \\
\text { al. }[83]\end{array}$ & Clodrolip or Zoledronic acid & - & Eliminating existent TAMs & Enhance the efficacy of sorafenib in mouse models \\
\hline $\begin{array}{l}\text { Zhou et } \\
\text { al.[117] }\end{array}$ & Zoledronic acid & - & Eliminating existent TAMs & Enhance the efficacy of TACE in HCC mouse models \\
\hline Tan et al. [95] & Baicalin & - & Re-educating TAMs & Suppress tumor growth \\
\hline Sun et al.[119] & 8-Bromo-7-methoxychrysin & CD163 & Re-educating TAMs & Impede interaction between HCC stem cells and TAMs \\
\hline Ao et al. ${ }^{[120]}$ & PLX3397 & CSF1R & Re-educating TAMs & Suppress tumor growth \\
\hline Wan et al.[87] & Tocilizumab & $\begin{array}{l}\text { IL-6 } \\
\text { receptor }\end{array}$ & Neutralizing productsof TAMs & Inhibit TAMs mediated stimulation of HCC stem cells \\
\hline
\end{tabular}

Table 2. Clinical trials targeting TAMs for HCC treatment

\begin{tabular}{|c|c|c|c|c|}
\hline Target & Agent & Mechanism & Phase & $\begin{array}{l}\text { Clinical trial } \\
\text { number }\end{array}$ \\
\hline Glypican-3 & GC33 & Glypican-3 antagonist (eliminating existent macrophages) & Phase 2 & NCT01507168 \\
\hline $\mathrm{CCR} 2 / 5$ & $\begin{array}{l}\text { Nivolumab } \\
\text { +BMS-813160 } \\
\text { /+BMS-986253 }\end{array}$ & CCR2/5 antagonist (inhibits monocyte/macrophage infiltration) & Phase 2 & NCT04123379 \\
\hline CSF1R & Chiauranib & $\begin{array}{l}\text { Multi-target inhibitor that suppresses angiogenesis-related kinases and CSF1R; decreases } \\
\text { the macrophage differentiation. }\end{array}$ & Phase 1 & NCT03245190 \\
\hline
\end{tabular}




\subsection{Therapies inhibiting the recruitment of macrophages}

The inhibition of monocyte recruitment in HCC tissues has recently emerged as a promising approach to decrease the levels of TAMs. Aberrant expression of miR-26a has been shown to suppress HCC growth and macrophage infiltration by targeting macrophage colony-stimulating factor (M-CSF) in a PI3K/AKT pathway-dependent mechanism [109]. CCL2 expression has been reported to be elevated in HCC tissues and has been suggested as a novel prognostic factor for HCC. Kuffer cells, as the primary source of CCL2, are essential for the recruitment and education of monocyte-derived macrophages [36]. The CCL2/CCR2 signaling has recently emerged as a target to suppress the recruitment of monocytes in tumors. Blockade of CCL2/CCR2 signaling pathway suppressed monocyte recruitment and the polarization of infiltrated macrophages towards the M2 phenotype, suppressing tumor growth in a $\mathrm{T}$ cell-dependent manner, in a mouse liver cancer model [110]. The CCR2 antagonist 747 has been shown to exert potent antitumor effects and enhance the efficacy of sorafenib by combating TAM-mediated immunosuppression and increasing the number of CD8+ T cells in an HCC mouse model [97]. Glypican-3 has been reported to be overexpressed in HCC and has been implicated in the recruitment of macrophages by binding to CCL3 and CCL5 [111,112]. Antibodies targeting glypican-3 have demonstrated promising efficacy in several clinical trials by inhibiting the recruitment of M2 macrophages in the TME [113]. For example, the humanized antibody GC33 was well tolerated in advanced HCC patients in a clinical trial conducted in Japan [114].

\subsection{Therapies eliminating pre-existing TAMs}

The combination of TAM-targeting therapies with traditional therapies in HCC has been investigated [21]. For instance, clodrolip or ZA treatment augmented the antitumor effects of sorafenib, by suppressing tumor growth, angiogenesis, and lung metastasis in HCC xenograft models [83]. The use of ZA may remodel the intratumoral pool of macrophages by trigging apoptosis in specific TAM populations $[115,116]$. In addition, ZA treatment has been shown to enhance the effects of transarterial chemoembolization by suppressing the infiltration of TAMs in HCC [117].

\subsection{Reprogramming TAM polarization}

Numerous tumor-derived factors are involved in TAM phenotype shift, including the aforementioned TGF- $\beta$, miR-98, and long non-coding RNA COX-2 $[66,70,71]$. Recently, it is reported that HCC-derived exosomes could remodel macrophages by activating NF-KB signaling and result in M2 polarized TAMs [54]. Receptor-interacting protein kinase 3 (RIPK3) is downregulated in HCC-associated macrophages, and RIPK3 deficiency induced fatty acid oxidation (FAO), which induced M2 polarized TAMs. Hence, RIPK3 upregulation or FAO blockade reversed the immunosuppressive activity of TAMs and dampened HCC tumorigenesis [118]. As mentioned previously, TAMs with the M1 phenotype promote tumor cell elimination and degradation. Hence, re-educating TAMs to switch from the M2 to M1 phenotype has been suggested as a therapeutic approach for HCC. Baicalin administration suppressed tumor growth in an orthotopic HCC mouse model by inducing TAM reprogramming towards the M1 phenotype and subsequent secretion of pro-inflammatory cytokines [95]. Furthermore, 8-bromo-7-methoxychrysin (BrMC) was shown to attenuate the effects of M2 macrophages by influencing the profile of secreted cytokines and reversing M2 polarization of TAMs [119]. The use of the competitive CSF-1R inhibitor PLX3397 suppressed tumor growth in an HCC mouse model by shifting the polarization of TAMs towards the M1 phenotype [120].

\section{Conclusions}

Macrophages play essential roles in orchestrating immune responses; nevertheless, dysregulation of their function has been implicated in HCC, among other solid malignancies. Macrophages are abundant in the TME in HCC, exhibiting double-edged roles in controlling tumorigenesis by modulating immune responses. Importantly, TAMs have been shown to enhance HCC development and progression by promoting immune suppression, cancer cell proliferation, invasion, metastasis, and maintenance of cancer cell stemness. Consequently, a better understanding of the mechanisms by which TAMs regulate HCC malignancy would allow for the development of novel and more effective TAM-targeting HCC therapies. Adjuvant treatment with agents targeting TAMs following conventional hepatectomy or liver transplantation may improve the clinical benefit of the therapies currently used for HCC patients.

\section{Abbreviations}

HCC: hepatocellular carcinoma; TACE: transarterial chemoembolization; RFA: radiofrequency ablation; PD-1: programmed cell death protein 1; CTLA-4: cytotoxic T lymphocyte associated antigen 4; TME: tumor microenvironment; TAM: tumor associated macrophage; DC: dendritic cell; MoMFs: monocyte-derived macrophages; LPS: 
lipopolysaccharides; IFN- $\gamma$ : interferon- $\gamma$; TNF: tumor necrosis factor; TLR: toll like receptor; NO: nitric oxide; ROS: reactive oxygen species; IL-1: interleukin 1; CXCL5: C-X-C motif chemokine ligand 5; CCL5: C-C motif chemokine ligand 5; CSF: colony stimulating factor; mtDNA: mitochondrial DNA; miRNAs: micro RNAs; circRNAs: cicular RNAs; TGF- $\beta$ : transforming growth factor beta; Tregs: regulatory T cells; IGF1: insuline-like growth factor; EMT: epithelial-mesenchymal transition; IDO: indoleamine-2,3-dioxygenase; HIF-1a: hypoxia inducible factor 1a; PD-L1: programmed cell death ligand 1; CXCR4: C-X-C motif chemokine receptor 4; ZA: zoledronic acid; CSC: cancer stem cell; HGF: hepatocyte growth factor; BrMC: 8-bromo-7methoxychrysin; iNOS: inducible nitric oxide synthase; Arg 1: arginine 1.

\section{Acknowledgements}

The English in this document has been checked by at least two professional editors, both native speakers of English. For a certificate, please see: http://www.textcheck.com/certificate/C3nkIQ.

\section{Funding}

This work is supported by the National Natural Science Foundation of China (No.81572307 and 81773096).

\section{Authors' Contributions}

Yu Huang and Wenhao Ge contributed eqaully to this work. Yu Huang and Wenhao Ge concepted and wrote the manuscript; Jiarong Zhou, Bingqiang Gao and Xiaohui Qian collected and assemblied the information; Weilin Wang proofread and revised the manuscript; all of the authors approved the final version to be published.

\section{Competing Interests}

The authors have declared that no competing interest exists.

\section{References}

1. Siegel RL, Miller KD. Cancer statistics, 2019. 2019; 69: 7-34.

2. Sun JY, Yin T, Zhang XY. Therapeutic advances for patients with intermediate hepatocellular carcinoma. J Cell Physiol. 2019; 234: 12116-21.

3. EASL-EORTC clinical practice guidelines: management of hepatocellular carcinoma. Journal of hepatology. 2012; 56: 908-43.

4. Kumar A, Acharya SK, Singh SP, Arora A, Dhiman RK, Aggarwal R, et al. 2019 Update of Indian National Association for Study of the Liver Consensus on Prevention, Diagnosis, and Management of Hepatocellular Carcinoma in India: The Puri II Recommendations. Journal of clinical and experimental hepatology. 2020; 10: 43-80.

5. Méndez-Blanco C, Fondevila F. Sorafenib resistance in hepatocarcinoma: role of hypoxia-inducible factors. Experimental \& Molecular Medicine. 2018; 50: $1-9$.

6. Hargadon KM, Johnson CE, Williams CJ. Immune checkpoint blockade therapy for cancer: An overview of FDA-approved immune checkpoint inhibitors. International immunopharmacology. 2018; 62: 29-39.

7. Newick K, O'Brien S, Moon E, Albelda SM. CAR T Cell Therapy for Solid Tumors. Annual review of medicine. 2017; 68: 139-52.
8. Wei SC, Duffy CR. Fundamental Mechanisms of Immune Checkpoint Blockade Therapy. Cancer Discovery. 2018; 8: 1069-86.

9. El-Khoueiry AB, Sangro B, Yau T, Crocenzi TS, Kudo M, Hsu C, et al. Nivolumab in patients with advanced hepatocellular carcinoma (CheckMate 040): an open-label, non-comparative, phase $1 / 2$ dose escalation and expansion trial. Lancet (London, England). 2017; 389: 2492-502.

10. Yang JD, Hainaut P, Gores GJ. A global view of hepatocellular carcinoma: trends, risk, prevention and management. Nat Rev Gastroenterol Hepatol. 2019; 16: 589-604.

11. Forner A, Llovet JM, Bruix J. Hepatocellular carcinoma. Lancet (London, England). 2012; 379: 1245-55.

12. Lu C, Rong D, Zhang B, Zheng W, Wang X, Chen Z, et al. Current perspectives on the immunosuppressive tumor microenvironment in hepatocellular carcinoma: challenges and opportunities. Molecular cancer. 2019; 18: 130

13. Fu Y, Liu S, Zeng S, Shen H. From bench to bed: the tumor immune microenvironment and current immunotherapeutic strategies for hepatocellular carcinoma. Journal of experimental \& clinical cancer research : CR. 2019; 38: 396.

14. Capece D, Fischietti M, Verzella D, Gaggiano A, Cicciarelli G, Tessitore A, et al. The inflammatory microenvironment in hepatocellular carcinoma: a pivotal role for tumor-associated macrophages. BioMed research international. 2013; 2013: 187204.

15. Hanahan D, Coussens LM. Accessories to the crime: functions of cells recruited to the tumor microenvironment. Cancer cell. 2012; 21: 309-22

16. Tian Z, Hou X, Liu W, Han Z, Wei L. Macrophages and hepatocellular carcinoma. Cell \& Bioscience. 2019; 9: 79 .

17. Xu W, Liu K, Chen M, Sun JY, McCaughan GW, Lu XJ, et al. Immunotherapy for hepatocellular carcinoma: recent advances and future perspectives. Ther Adv Med. Oncol. 2019; 11: 1758835919862692.

18. Gun SY, Lee SWL, Sieow JL, Wong SC. Targeting immune cells for cancer therapy. Redox biology. 2019; 25: 101174.

19. Okusaka T, Ikeda M. Immunotherapy for hepatocellular carcinoma: current status and future perspectives. ESMO open. 2018; 3: e000455.

20. Cassetta L, Pollard JW. Targeting macrophages: therapeutic approaches in cancer. Nature reviews Drug discovery. 2018; 17: 887-904.

21. Ruffell B, Coussens LM. Macrophages and therapeutic resistance in cancer. Cancer cell. 2015; 27: 462-72.

22. Galdiero MR, Bonavita E, Barajon I, Garlanda C, Mantovani A, Jaillon S. Tumor associated macrophages and neutrophils in cancer. Immunobiology. 2013; 218: 1402-10.

23. Noy R, Pollard JW. Tumor-associated macrophages: from mechanisms to therapy. Immunity. 2014; 41: 49-61.

24. Fu XT, Song K, Zhou J, Shi YH, Liu WR, Shi GM, et al. Tumor-associated macrophages modulate resistance to oxaliplatin via inducing autophagy in hepatocellular carcinoma. Cancer cell international. 2019; 19:71.

25. Dong N, Shi X, Wang S, Gao Y, Kuang Z, Xie Q, et al. M2 macrophages mediate sorafenib resistance by secreting HGF in a feed-forward manner in hepatocellular carcinoma. Br J Cancer. 2019; 121: 22-33.

26. Kim J, Bae JS. Tumor-Associated Macrophages and Neutrophils in Tumor Microenvironment. Mediators of inflammation. 2016; 2016: 6058147.

27. Clappaert EJ, Murgaski A, Van Damme H, Kiss M, Laoui D. Diamonds in the Rough: Harnessing Tumor-Associated Myeloid Cells for Cancer Therapy. Frontiers in immunology. 2018; 9: 2250.

28. Petty AJ, Yang Y. Tumor-associated macrophages: implications in cancer immunotherapy. Immunotherapy. 2017; 9: 289-302

29. Tacke F. Targeting hepatic macrophages to treat liver diseases. Journal of hepatology. 2017; 66: 1300-12.

30. Wynn TA, Chawla A, Pollard JW. Macrophage biology in development, homeostasis and disease. Nature. 2013: 496: 445-55.

31. Gordon S, Plüddemann A, Martinez Estrada F. Macrophage heterogeneity in tissues: phenotypic diversity and functions. Immunological reviews. 2014; 262: 36-55.

32. Heymann F, Tacke F. Immunology in the liver--from homeostasis to disease. Nature reviews Gastroenterology \& hepatology. 2016; 13: 88-110.

33. Vannella KM, Wynn TA. Mechanisms of Organ Injury and Repair by Macrophages. Annual review of physiology. 2017; 79: 593-617.

34. Degroote H, Van Dierendonck A, Geerts A, Van Vlierberghe H, Devisscher L. Preclinical and Clinical Therapeutic Strategies Affecting Tumor-Associated Macrophages in Hepatocellular Carcinoma. J of Immunology Research. 2018; 2018: 7819520

35. Krenkel O, Tacke F. Liver macrophages in tissue homeostasis and disease. Nature reviews Immunology. 2017; 17: 306-21.

36. Okabe Y, Medzhitov R. Tissue biology perspective on macrophages. Nature immunology. 2016; 17: 9-17

37. Gordon S, Taylor PR. Monocyte and macrophage heterogeneity. Nature reviews Immunology. 2005; 5: 953-64.

38. Sica A, Erreni M, Allavena P, Porta C. Macrophage polarization in pathology. Cellular and molecular life sciences : CMLS. 2015; 72: 4111-26.

39. Galli SJ, Borregaard N, Wynn TA. Phenotypic and functional plasticity of cells of innate immunity: macrophages, mast cells and neutrophils. Nature immunology. 2011; 12: 1035-44.

40. Chawla A, Nguyen KD, Goh YP. Macrophage-mediated inflammation in metabolic disease. Nature reviews Immunology. 2011; 11: 738-49. 
41. Sica A, Invernizzi P, Mantovani A. Macrophage plasticity and polarization in liver homeostasis and pathology. Hepatology (Baltimore, Md). 2014; 59: 2034-42.

42. Dou L, Shi X, He X, Gao Y. Macrophage Phenotype and Function in Liver Disorder. Frontiers in immunology. 2019; 10: 3112.

43. Li XY, Yang X, Zhao QD, Han ZP, Liang L, Pan XR, et al. Lipopolysaccharide promotes tumorigenicity of hepatic progenitor cells by promoting proliferation and blocking normal differentiation. Cancer letters. 2017; 386: $35-46$

44. Yu LX, Ling Y, Wang HY. Role of nonresolving inflammation in hepatocellular carcinoma development and progression. NPJ precision oncology. 2018; 2: 6.

45. Elliott LA, Doherty GA, Sheahan K, Ryan EJ. Human Tumor-Infiltrating Myeloid Cells: Phenotypic and Functional Diversity. Frontiers in immunology. 2017; 8: 86.

46. Dong P, Ma L, Liu L, Zhao G, Zhang S, Dong L, et al. CD86 ${ }^{+} / \mathrm{CD}_{206}$, Diametrically Polarized Tumor-Associated Macrophages, Predict Hepatocellular Carcinoma Patient Prognosis. International journal of molecular sciences. 2016; 17: 320.

47. Bonavita E, Gentile S, Rubino M, Maina V, Papait R, Kunderfranco P, et al. PTX3 is an extrinsic oncosuppressor regulating complement-dependent inflammation in cancer. Cell. 2015; 160: 700-14.

48. Weitzenfeld P, Ben-Baruch A. The chemokine system, and its CCR5 and CXCR4 receptors, as potential targets for personalized therapy in cancer. Cancer letters. 2014; 352: 36-53.

49. Pollard JW. Tumour-educated macrophages promote tumour progression and metastasis. Nature reviews Cancer. 2004; 4: 71-8.

50. Kong L, Zhou Y, Bu H, Lv T, Shi Y, Yang J. Deletion of interleukin-6 in monocytes/macrophages suppresses the initiation of hepatocellular carcinoma in mice. Journal of experimental \& clinical cancer research : CR. 2016; 35: 131.

51. Tymoszuk P, Evens H, Marzola V, Wachowicz K, Wasmer MH, Datta S, et al. In situ proliferation contributes to accumulation of tumor-associated macrophages in spontaneous mammary tumors. European journal of immunology. 2014; 44: 2247-62.

52. Bao D, Zhao J, Zhou X, Yang Q, Chen Y, Zhu J, et al. Mitochondrial fission-induced mtDNA stress promotes tumor-associated macrophage infiltration and HCC progression. Oncogene. 2019; 38: 5007-20.

53. Zhu Y, Yang J, Xu D, Gao XM, Zhang Z, Hsu JL, et al. Disruption of tumour-associated macrophage trafficking by the osteopontin-induced colony-stimulating factor-1 signalling sensitises hepatocellular carcinoma to anti-PD-L1 blockade. Gut. 2019; 68: 1653-66.

54. Yin C, Han Q, Xu D, Zheng B, Zhao X, Zhang J. SALL4-mediated upregulation of exosomal miR-146a-5p drives T-cell exhaustion by M2 tumor-associated macrophages in HCC. Oncoimmunology. 2019; 8: 1601479.

55. Hu ZQ, Zhou SL. Circular RNA Sequencing Identifies CircASAP1 as a Key Regulator in Hepatocellular Carcinoma Metastasis. Hepatology. 2020; 72(3):906-922.

56. Ravà M, D'Andrea A, Doni M, Kress TR, Ostuni R, Bianchi V, et al. Mutual epithelium-macrophage dependency in liver carcinogenesis mediated by ST18. Hepatology (Baltimore, Md). 2017; 65: 1708-19.

57. Wang D, Yang L, Yue D, Cao L, Li L, Wang D, et al. Macrophage-derived CCL22 promotes an immunosuppressive tumor microenvironment via IL-8 in malignant pleural effusion. Cancer letters. 2019; 452: 244-53.

58. Bekkering S, Arts RJW, Novakovic B, Kourtzelis I, van der Heijden C, Li Y, et al. Metabolic Induction of Trained Immunity through the Mevalonate Pathway. Cell. 2018; 172: 135-46.e9.

59. Saeed S, Quintin J, Kerstens HH, Rao NA, Aghajanirefah A, Matarese F, et al. Epigenetic programming of monocyte-to-macrophage differentiation and trained innate immunity. Science (New York, NY). 2014; 345: 1251086.

60. Quail DF, Bowman RL, Akkari L, Quick ML, Schuhmacher AJ, Huse JT, et al. The tumor microenvironment underlies acquired resistance to CSF-1R inhibition in gliomas. Science (New York, NY). 2016; 352: aad3018.

61. Thorsson V, Gibbs DL, Brown SD, Wolf D, Bortone DS, Ou Yang TH, et al. The Immune Landscape of Cancer. Immunity. 2019; 51: 411-2.

62. Chew V, Lai L, Pan L, Lim CJ, Li J, Ong R, et al. Delineation of an immunosuppressive gradient in hepatocellular carcinoma using high-dimensional proteomic and transcriptomic analyses. Proceedings of the National Academy of Sciences of the United States of America. 2017; 114: E5900-e9.

63. Yao RR, Li JH, Zhang R, Chen RX, Wang YH. M2-polarized tumor-associated macrophages facilitated migration and epithelial-mesenchymal transition of HCC cells via the TLR4/STAT3 signaling pathway. World journal of surgical oncology. 2018; 16: 9

64. Xiao P, Long X, Zhang L, Ye Y, Guo J. Neurotensin/IL-8 pathway orchestrates local inflammatory response and tumor invasion by inducing M2 polarization of Tumor-Associated macrophages and epithelial-mesenchymal transition of hepatocellular carcinoma cells. Oncoimmunology. 2018; 7(7): e1440166.

65. Yin Z, Huang J, Ma T, Li D, Wu Z, Hou B, et al. Macrophages activating chemokine (C-X-C motif) ligand 8/miR-17 cluster modulate hepatocellular carcinoma cell growth and metastasis. American journal of translational research. 2017; 9: 2403-11.

66. Yan W, Liu X, Ma H. Tim-3 fosters HCC development by enhancing TGF- $\beta$-mediated alternative activation of macrophages. Gut. 2015; 64(10): 1593-604.
67. Jiang J, Wang GZ, Wang Y, Huang HZ, Li WT, Qu XD. Hypoxia-induced HMGB1 expression of HCC promotes tumor invasiveness and metastasis via regulating macrophage-derived IL-6. Experimental cell research. 2018; 367: 81-8.

68. Zhang YL, Li Q, Yang XM, Fang F, Li J, Wang YH, et al. SPON2 Promotes M1-like Macrophage Recruitment and Inhibits Hepatocellular Carcinoma Metastasis by Distinct Integrin-Rho GTPase-Hippo Pathways. Cancer research. 2018; 78: 2305-17.

69. Zhou SL, Hu ZQ, Zhou ZJ, Dai Z, Wang Z, Cao Y, et al. miR-28-5p-IL-34-macrophage feedback loop modulates hepatocellular carcinoma metastasis. Hepatology (Baltimore, Md). 2016; 63: 1560-75.

70. Li L, Sun P, Zhang C, Li Z, Cui K, Zhou W. MiR-98 modulates macrophage polarization and suppresses the effects of tumor-associated macrophages on promoting invasion and epithelial-mesenchymal transition of hepatocellular carcinoma. Cancer cell international. 2018; 18: 95.

71. Ye Y, Xu Y, Lai Y, He W, Li Y, Wang R, et al. Long non-coding RNA cox-2 prevents immune evasion and metastasis of hepatocellular carcinoma by altering M1/M2 macrophage polarization. J Cell Biochem. 2018; 119: 2951-63.

72. Tan S, Zhao J, Sun Z, Cao S, Niu K, Zhong Y, et al. Hepatocyte-specific TAK1 deficiency drives RIPK1 kinase-dependent inflammation to promote liver fibrosis and hepatocellular carcinoma. PNAS. 2020; 117: 14231-42.

73. Wu J, Gao W, Tang Q, Yu Y, You W, Wu Z, et al. M2 macrophage-derived exosomes facilitate hepatocarcinoma metastasis by transferring $\alpha(M) \beta(2)$ integrin to tumor cells. Hepatology (Baltimore, Md). 2020.

74. Yang Y, Ye YC. Crosstalk between hepatic tumor cells and macrophages via Wnt/ $\beta$-catenin signaling promotes M2-like macrophage polarization and reinforces tumor malignant behaviors. Cell Death \& Disease. 2018; 9: 793.

75. Liu HT, Huang DA, Li MM, Liu HD, Guo K. HSF1: a mediator in metabolic alteration of hepatocellular carcinoma cells in cross-talking with tumor-associated macrophages. American journal of translational research. 2019; 11: 5054-64.

76. Ye LY, Chen $\mathrm{W}$, Bai XL, Xu XY, Zhang Q, Xia XF, et al. Hypoxia-Induced Epithelial-to-Mesenchymal Transition in Hepatocellular Carcinoma Induces an Immunosuppressive Tumor Microenvironment to Promote Metastasis. Cancer research. 2016; 76: 818-30

77. Zhang J, Zhang Q, Lou Y, Fu Q, Chen Q, Wei T, et al. Hypoxia-inducible factor-1 $\alpha /$ interleukin-1 $\beta$ signaling enhances hepatoma epithelial-mesenchymal transition through macrophages in a hypoxicinflammatory microenvironment. Hepatology. 2018; 67: 1872-89.

78. Wu Q, Zhou W, Yin S, Zhou Y, Chen T, Qian J, et al. Blocking Triggering Receptor Expressed on Myeloid Cells-1-Positive Tumor-Associated Macrophages Induced by Hypoxia Reverses Immunosuppression and Anti-Programmed Cell Death Ligand 1 Resistance in Liver Cancer. Hepatology. 2019; 70: 198-214.

79. Deryugina EI, Quigley JP. Tumor angiogenesis: MMP-mediated induction of intravasation- and metastasis-sustaining neovasculature. Matrix biology : journal of the International Society for Matrix Biology. 2015; 44-46: 94-112.

80. Bartneck M, Schrammen PL, Möckel D, Govaere O, Liepelt A, Krenkel O, et al. The CCR2(+) Macrophage Subset Promotes Pathogenic Angiogenesis for Tumor Vascularization in Fibrotic Livers. Cellular and molecular gastroenterology and hepatology. 2019; 7: 371-90.

81. Zang M, Li Y, He H, Ding H, Chen K, Du J, et al. IL-23 production of liver inflammatory macrophages to damaged hepatocytes promotes hepatocellular carcinoma development after chronic hepatitis B virus infection. Biochimica et biophysica acta Molecular basis of disease. 2018; 1864: 3759-70.

82. Meng $\mathrm{YM}$, Liang $\mathrm{J}, \mathrm{Wu} \mathrm{C}, \mathrm{Xu} \mathrm{J}$, Zeng $\mathrm{DN}, \mathrm{Yu} \mathrm{XJ}$, et al. Monocytes/Macrophages promote vascular CXCR4 expression via the ERK pathway in hepatocellular carcinoma. Oncoimmunology. 2018; 7: e1408745.

83. Zhang W, Zhu XD, Sun HC, Xiong YQ, Zhuang PY, Xu HX, et al. Depletion of tumor-associated macrophages enhances the effect of sorafenib in metastatic liver cancer models by antimetastatic and antiangiogenic effects. Clinical cancer research : an official journal of the American Association for Cancer Research. 2010; 16: 3420-30.

84. Ma S, Chan KW, Hu L, Lee TK, Wo JY, Ng IO, et al. Identification and characterization of tumorigenic liver cancer stem/progenitor cells. Gastroenterology. 2007; 132: 2542-56.

85. Nio K, Yamashita T, Okada H, Kondo M, Hayashi T, Hara Y, et al. Defeating $\operatorname{EpCAM}(+)$ liver cancer stem cells by targeting chromatin remodeling enzyme CHD4 in human hepatocellular carcinoma. Journal of hepatology. 2015; 63: 1164-72.

86. Kopanja D, Pandey A, Kiefer M, Wang Z, Chandan N, Carr JR, et al. Essential roles of FoxM1 in Ras-induced liver cancer progression and in cancer cells with stem cell features. Journal of hepatology. 2015; 63: 429-36.

87. Wan S, Zhao E, Kryczek I, Vatan L, Sadovskaya A, Ludema G, et al. Tumor-associated macrophages produce interleukin 6 and signal via STAT3 to promote expansion of human hepatocellular carcinoma stem cells. Gastroenterology. 2014; 147: 1393-404.

88. Fan QM, Jing YY, Yu GF, Kou XR, Ye F, Gao L, et al. Tumor-associated macrophages promote cancer stem cell-like properties via transforming growth factor-beta1-induced epithelial-mesenchymal transition in hepatocellular carcinoma. Cancer letters. 2014; 352: 160-8.

89. Chen $\mathrm{Y}$, Wen $\mathrm{H}$, Zhou C, Su Q, Lin Y, Xie Y, et al. TNF-a derived from M2 tumor-associated macrophages promotes epithelial-mesenchymal transition and cancer stemness through the $\mathrm{Wnt} / \beta$-catenin pathway in SMMC-7721 hepatocellular carcinoma cells. Experimental cell research. 2019; 378: 41-50. 
90. Wang Y, Wang B, Xiao S, Li Y, Chen Q. miR-125a/b inhibits tumor-associated macrophages mediated in cancer stem cells of hepatocellular carcinoma by targeting CD90. J Cell Biochem. 2019; 120: 3046-55.

91. Ngabire D, Kim GD. Autophagy and Inflammatory Response in the Tumor Microenvironment. International journal of molecular sciences. Int J Mol Sci. 2017; 18(9).

92. Sun $\mathrm{K}$, Xu L, Jing Y, Han Z, Chen X, Cai C, et al. Autophagy-deficient Kupffer cells promote tumorigenesis by enhancing mtROS-NF-kB-IL1 $\alpha / \beta$-dependent inflammation and fibrosis during the preneoplastic stage of hepatocarcinogenesis. Cancer letters. 2017; 388: 198-207.

93. Chang CP, Su YC, Lee PH, Lei HY. Targeting NFKB by autophagy to polarize hepatoma-associated macrophage differentiation. Autophagy. 2013; 9: 619-21.

94. Lin H, Yan J, Wang Z, Hua F, Yu J, Sun W, et al. Loss of immunity-supported senescence enhances susceptibility to hepatocellular carcinogenesis and progression in Toll-like receptor 2-deficient mice. Hepatology (Baltimore, Md). 2013; 57: 171-82.

95. Tan HY, Wang N, Man K, Tsao SW, Che CM, Feng Y. Autophagy-induced RelB/p52 activation mediates tumour-associated macrophage repolarisation and suppression of hepatocellular carcinoma by natural compound baicalin. Cell death \& disease. 2015; 6: e1942.

96. Xia S, Pan Y, Liang Y, Xu J, Cai X. The microenvironmental and metabolic aspects of sorafenib resistance in hepatocellular carcinoma. EBioMedicine. 2020; 51: 102610

97. Yao W, Ba O, Li X, Li H, Zhang S, Yuan Y, et al. A Natural CCR2 Antagonist Relieves Tumor-associated Macrophage-mediated Immunosuppression to Produce a Therapeutic Effect for Liver Cancer. EBioMedicine. 2017; 22: 58-67.

98. Zhou SL, Zhou ZJ, Hu ZQ, Huang XW, Wang Z, Chen EB, et al. Tumor-Associated Neutrophils Recruit Macrophages and T-Regulatory Cells to Promote Progression of Hepatocellular Carcinoma and Resistance to Sorafenib. Gastroenterology. 2016; 150: 1646-58.e17.

99. Hage C, Hoves S, Strauss L, Bissinger S, Prinz Y, Pöschinger T et al Sorafenib Induces Pyroptosis in Macrophages and Triggers Natural Killer Cell-Mediated Cytotoxicity Against Hepatocellular Carcinoma. Hepatology (Baltimore, Md). 2019; 70: 1280-97.

100. Mantovani A, Marchesi F, Malesci A, Laghi L, Allavena P. Tumour-associated macrophages as treatment targets in oncology. Nature reviews Clinical oncology. 2017; 14: 399-416.

101. Tang X, Mo C, Wang Y, Wei D, Xiao H. Anti-tumour strategies aiming to target tumour-associated macrophages. Immunology. 2013; 138: 93-104.

102. Jaiswal S, Jamieson CH, Pang WW, Park CY, Chao MP, Majeti R, et al. CD47 is upregulated on circulating hematopoietic stem cells and leukemia cells to avoid phagocytosis. Cell. 2009; 138: 271-85.

103. Tseng D, Volkmer JP, Willingham SB, Contreras-Trujillo H, Fathman JW, Fernhoff NB, et al. Anti-CD47 antibody-mediated phagocytosis of cancer by macrophages primes an effective antitumor T-cell response. Proceedings of the National Academy of Sciences of the United States of America. 2013; 110: 11103-8.

104. Okazawa H, Motegi S, Ohyama N, Ohnishi H, Tomizawa T, Kaneko Y, et al. Negative regulation of phagocytosis in macrophages by the CD47-SHPS-1 system. Journal of immunology (Baltimore, Md : 1950). 2005; 174: 2004-11.

105. Chen J, Zheng DX, Yu XJ, Sun HW, Xu YT, Zhang YJ, et al. Macrophages induce CD47 upregulation via IL-6 and correlate with poor survival in hepatocellular carcinoma patients. Oncoimmunology. 2019; 8: e1652540.

106. Yang HD, Kim HS, Kim SY, Na MJ, Yang G, Eun JW, et al. HDAC6 Suppresses Let-7i-5p to Elicit TSP1/CD47-Mediated Anti-Tumorigenesis and Phagocytosis of Hepatocellular Carcinoma. Hepatology (Baltimore, Md). 2019; 70: $1262-79$.

107. Lo J, Lau EY, So FT, Lu P, Chan VS, Cheung VC, et al. Anti-CD47 antibody suppresses tumour growth and augments the effect of chemotherapy treatment in hepatocellular carcinoma. Liver international. 2016; 36: 737-45.

108. Xiao Z, Chung H, Banan B, Manning PT, Ott KC, Lin S, et al. Antibody mediated therapy targeting CD47 inhibits tumor progression of hepatocellular carcinoma. Cancer letters. 2015; 360: 302-9.

109. Chai ZT, Zhu XD, Ao JY, Wang WQ, Gao DM, Kong J, et al. microRNA-26a suppresses recruitment of macrophages by down-regulating macrophage colony-stimulating factor expression through the PI3K/Akt pathway in hepatocellular carcinoma. Journal of hematology \& oncology. 2015; 8: 56.

110. Li X, Yao W, Yuan Y, Chen P, Li B, Li J, et al. Targeting of tumour-infiltrating macrophages via CCL2/CCR2 signalling as a therapeutic strategy against hepatocellular carcinoma. Gut. 2017; 66: 157-67.

111. Yao M, Wang L, Dong Z, Qian Q, Shi Y, Yu D, et al. Glypican-3 as an emerging molecular target for hepatocellular carcinoma gene therapy. Tumour biology. 2014; 35: 5857-68.

112. Takai H, Ashihara M, Ishiguro T, Terashima H, Watanabe $T$, Kato A, et al. Involvement of glypican-3 in the recruitment of M2-polarized tumor-associated macrophages in hepatocellular carcinoma. Cancer biology \& therapy. 2009; 8: 2329-38

113. Zhu AX, Gold PJ, El-Khoueiry AB, Abrams TA, Morikawa H, Ohishi N, et al. First-in-man phase I study of GC33, a novel recombinant humanized antibody against glypican-3, in patients with advanced hepatocellular carcinoma. Clinical cancer research. 2013; 19: 920-8.

114. Ikeda M, Ohkawa S, Okusaka T, Mitsunaga S, Kobayashi S, Morizane C, et al. Japanese phase I study of GC33, a humanized antibody against glypican-3 for advanced hepatocellular carcinoma. Cancer science. 2014; 105: 455-62.
115. Rogers TL, Wind N, Hughes R, Nutter F, Brown HK, Vasiliadou I, et al. Macrophages as potential targets for zoledronic acid outside the skeleton-evidence from in vitro and in vivo models. Cellular oncology (Dordrecht). 2013; 36: 505-14.

116. Coscia M, Quaglino E, Iezzi M, Curcio C, Pantaleoni F, Riganti C, et al. Zoledronic acid repolarizes tumour-associated macrophages and inhibits mammary carcinogenesis by targeting the mevalonate pathway. Journal of cellular and molecular medicine. 2010; 14: 2803-15.

117. Zhou DY, Qin J, Huang J, Wang F, Xu GP, Lv YT, et al. Zoledronic acid inhibits infiltration of tumor-associated macrophages and angiogenesis following transcatheter arterial chemoembolization in rat hepatocellular carcinoma models. Oncology letters. 2017; 14: 4078-84

118. Wu L, Zhang X, Zheng L, Zhao H, Yan G, Zhang Q, et al. RIPK3 Orchestrates Fatty Acid Metabolism in Tumor-Associated Macrophages and Hepatocarcinogenesis. Cancer Immunology Research. 2020; 8: 710-21.

119. Sun S, Cui Y, Ren K, Quan M, Song Z, Zou H, et al. 8-bromo-7-methoxychrysin Reversed M2 Polarization of Tumor-associated Macrophages Induced by Liver Cancer Stem-like Cells. Anti-cancer agents in medicinal chemistry. 2017; 17: $286-93$

120. Ao JY, Zhu XD, Chai ZT, Cai H, Zhang YY, Zhang $K Z$, et al. Colony-Stimulating Factor 1 Receptor Blockade Inhibits Tumor Growth by Altering the Polarization of Tumor-Associated Macrophages in Hepatocellular Carcinoma. Molecular cancer therapeutics. 2017; 16: 1544-54. 